\title{
Haemodynamic, Hormonal and Urinary Responses to Postural Change in Tetraplegic and Paraplegic Man
}

\author{
J. S. Kooner, MB, MRCP ${ }^{1}$ H. L. Frankel, MB, FRCP, ${ }^{2}$ N. Mirando, \\ MB ${ }^{2}$ W. S. Pearî, FRCP, FRS, ${ }^{1}$ C. J. Mathias, DPhil, FRCP ${ }^{1}$ \\ ${ }^{1}$ Medical Unit, St Mary's Hospital Medical School, Norfolk Place, London, UK. \\ ${ }^{2}$ National Spinal Injuries Unit, Stoke Mandeville Hospital, Aylesbury, UK.
}

\begin{abstract}
Summary
We have studied the haemodynamic, hormonal and urinary effects of postural change in 6 tetraplegic patients, 6 paraplegic patients and 6 normal subjects. Measurements of blood pressure and heart rate, plasma renin activity, plasma aldosterone, urine volume and electrolyte excretion were made for 60 minutes while sitting and 60 minutes while recumbent. In tetraplegics the blood pressure was lower when sitting and rose during recumbency, unlike paraplegics and normal subjects. Plasma renin activity and aldosterone were higher in tetraplegics when sitting compared to normal subjects and did not fall during recumbency. Urine output increased significantly after recumbency in tetraplegics, but not in paraplegics or normal subjects. Both urinary sodium and potassium excretion were lower in tetraplegics and higher in paraplegics compared to normal subjects when sitting. In paraplegics the fall in both sodium and potassium excretion did not appear to be related to change in posture. Our observations indicate that recumbency induces a diuresis in tetraplegics but not in paraplegics or in normal subjects. The diuresis in tetraplegics may be related to the accompanying haemodynamic and hormonal changes induced by recumbency.
\end{abstract}

Key words: Tetraplegia; Diuresis; Recumbency; Haemodynamic aspects; Hormonal aspects.

Patients with chronic autonomic failure and postural hypotension have substantial nocturnal polyuria and overnight weight loss which probably reduces extracellular fluid volume and contributes to low morning blood pressure (Mathias et al., 1986). Tetraplegics also have sympathetic autonomic dysfunction, although the lesion is more specific and spinal sympathetic activity can be induced. We have investigated the effects of posture alone on cardiovascular, hormonal and urinary changes in a group of tetraplegics. Comparisons have been made with paraplegics with lesions below L1. These patients have an almost intact sympathetic nervous system but have reduced mobility. Normal subjects have been studied as controls. 


\section{Patients and methods}

We investigated 6 tetraplegics with chronic and complete cervical spinal cord transection (levels C4-C8), 6 paraplegics with complete lesions (levels T12-L3) and 6 normal subjects as controls. All subjects were on an unrestricted sodium diet and off drugs; previous medication was not thought to interfere with salt and water homeostasis. To ensure an adequate urine flow, a water load (15 $\mathrm{mls} / \mathrm{Kg}$ ) was given, followed by water replacement equal to the volume of urine passed. After three 30 minute periods for equilibration, the subjects were studied while sitting for 1 hour and then recumbent for 1 hour. Non invasive measurements of blood pressure and heart rate (Sentron) were made at 10 minute intervals. Blood samples for plasma renin activity and aldosterone (both radioimmunoassay) were taken from an indwelling venous cannula. Urine was collected at 30 minute intervals for measurements of volume, sodium, potassium and osmolality. All tetraplegics and paraplegics had urinary catheters. Tapping of the anterior abdominal wall and suprapubic compression were performed to ensure bladder emptying.

\section{Results}

In tetraplegics blood pressure was lower than normal subjects when sitting and rose during recumbency $(101 \pm 3 / 58 \pm 4$ to $109 \pm 3 / 64 \pm 4 \mathrm{mmHg}, \mathrm{p}<0.05)$. Blood pressure did not change after recumbency in normal subjects (132 $\pm /$ $81 \pm 6$ to $126 \pm 6 / 75 \pm 5 \mathrm{mmHg}$, not significant, NS) or paraplegics ( $125 \pm 5 /$ $74 \pm 5$ to $124 \pm 7 / 68 \pm 4 \mathrm{mmHg}$, NS). The recumbent blood pressure in tetraplegics was lower compared to normal subjects. Mean heart rate fell in all three groups after recumbency (Table I).

Plasma renin activity and aldosterone levels were higher when sitting in both tetraplegics and paraplegics compared to normal subjects. Both levels fell significantly in normal subjects only. Urine output was lower in tetraplegics when sitting compared to the other two groups.

Recumbency induced a significant increase in urine output in tetraplegics. There were no significant changes in both normal subjects or in paraplegics. In tetraplegics sodium excretion was lower than in normal subjects when sitting. After recumbency there was no change in sodium excretion in both tetraplegics or normal subjects. In paraplegics sodium excretion was much higher than in the other two groups when sitting and fell during recumbency (Table II). Sodium excretion continued to fall on assuming the sitting position. Potassium excretion too was lower in tetraplegics compared to normal subjects when sitting. After recumbency however potassium excretion rose in tetraplegics but not in normal subjects. In paraplegics as with sodium, potassium excretion was increased initially, fell during recumbency and fell further during repeat sitting at the end of the study. Urine osmolality was high in both tetraplegics and paraplegics when sitting compared to normal subjects. After recumbency the osmolality did not change in tetraplegics and normal subjects. In paraplegics osmolality was much higher than in both tetraplegics or normal subjects when sitting; there was a fall in osmolality during recumbency with a further fall on sitting again. Statistical analysis was performed using analysis of variance. If the null hypothesis was rejected, Scheff's comparisons were made, $\mathrm{p}<0.05$ was considered significant. 
Table I Pla sma and urinary changes (means \pm SEM) in normals, tetraplegics and pa raplegics with postural change

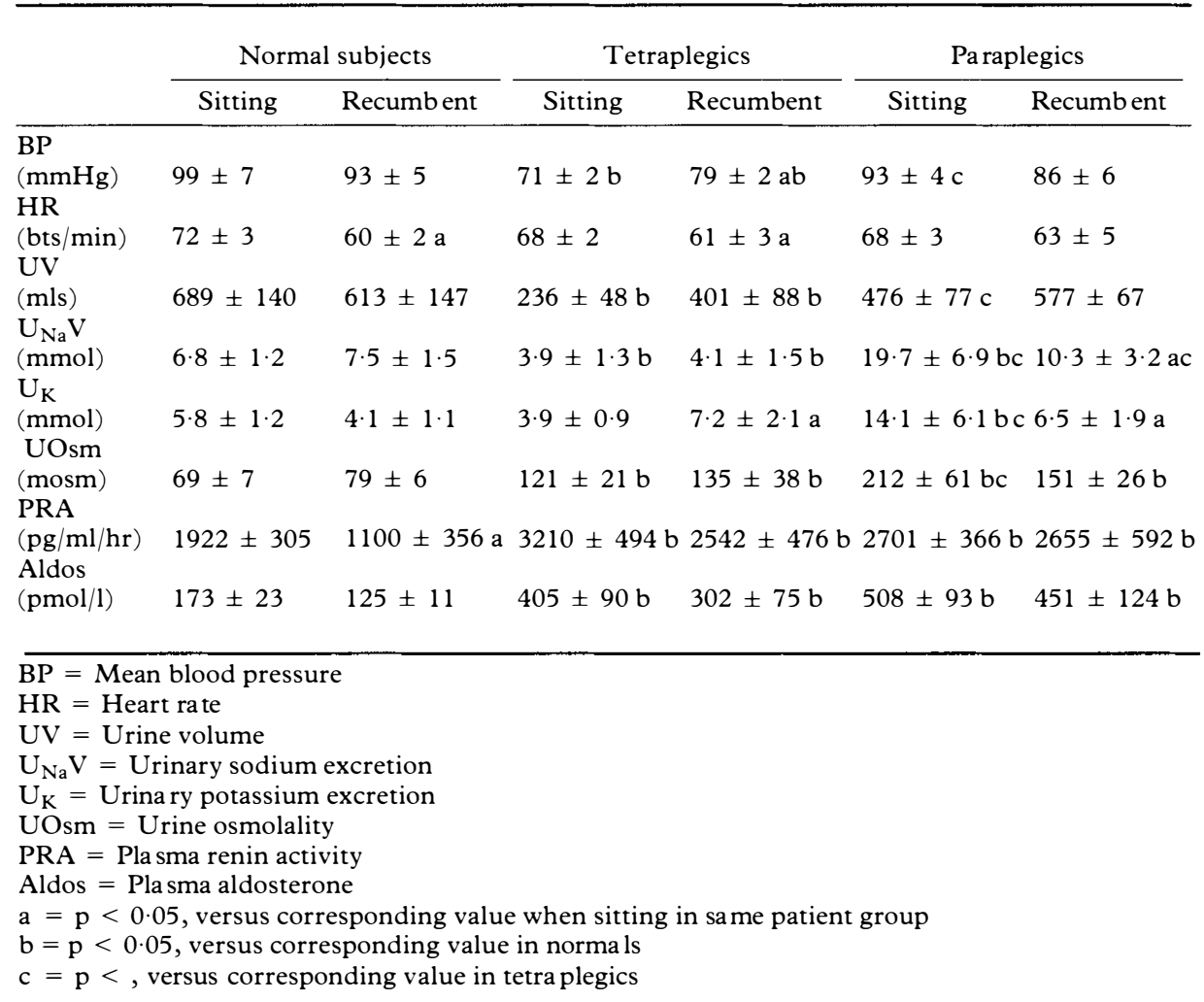

Table II Urinary changes (means \pm SEM) in pa raplegics with posture

\begin{tabular}{lccccc}
\hline & \multicolumn{5}{c}{ Pa raplegics } \\
\cline { 2 - 6 } & Sitting & Sitting & Recumbent & Recumbent & Sitting \\
\hline Time $(\min )$ & $0-30$ & $30-60$ & $60-90$ & $90-120$ & $120-150$ \\
$\mathrm{UV}(\mathrm{mls})$ & $233 \pm 34$ & $243 \pm 43$ & $333 \pm 41$ & $244 \pm 86$ & $292 \pm 84$ \\
$\mathrm{U}_{\mathrm{Na}}(\mathrm{mmol})$ & $12 \cdot 3 \pm 4 \cdot 2$ & $7 \cdot 5 \pm 2 \cdot 7$ & $6 \cdot 6 \pm 2 \cdot 1 \mathrm{a}$ & $3 \cdot 7 \pm 1 \cdot 6 \mathrm{a}$ & $3 \cdot 7 \pm 1 \cdot 3 \mathrm{ab}$ \\
$\mathrm{U}_{\mathrm{K}}(\mathrm{mmol})$ & $8 \cdot 1 \pm 2 \cdot 1$ & $6 \cdot 1 \pm 2 \cdot 0$ & $3 \cdot 0 \pm 0 \cdot 4 \mathrm{a}$ & $3 \cdot 5 \pm 1 \cdot 4 \mathrm{a}$ & $1 \cdot 8 \pm 0 \cdot 5 \mathrm{ab}$ \\
$\mathrm{UOsm}(\mathrm{mosm} / \mathrm{Kg})$ & $237 \pm 63$ & $191 \pm 44$ & $169 \pm 26$ & $133 \pm 25 \mathrm{a}$ & $119 \pm 22 \mathrm{a}$ \\
\hline
\end{tabular}

$\mathrm{UV}=$ Urine volume

$\mathrm{U}_{\mathrm{Na}} \mathrm{V}=$ Urinary sodium excretion

$\mathrm{U}_{\mathrm{K}} \mathrm{Urinary}$ potassium excretion

UOsm $=$ Urine osmola lity

$a=p<0.05$, versus corresponding value in first 30 min sitting period

$\mathrm{b}=\mathrm{p}<0.05$, versus corresponding value in first $30 \mathrm{~min}$ recumbent period

\section{Discussion}

Blood pressure was low in tetraplegics when sitting and rose after recumbency; this did not occur in normal subjects and paraplegics. The fall in blood pressure on sitting in tetraplegics is related to the inability of these patients to increase sympathetic activity in resonse to head-up postural change (Mathias et al., 1975). The rise in blood pressure during recumbency may be related to fluid shifts into the central compartment with an increase in venous return and stroke 
volume. Plasma renin activity and aldosterone were elevated in tetraplegics and paraplegics. In tetraplegics reduced systemic and renal perfusion pressure with afferent arteriolar dilation may result in stimulation of justaglomerular cells and higher renin and thus angiotensin II levels (Mathias et al., 1975, 1980). Higher levels of aldosterone in tetraplegics may result from angiotensin II mediated stimulation of the adrenal cortex.

In tetraplegics renin and aldosterone levels fell, but not significantly, during recumbency. This may reflect the long half life of renin and aldosterone as our measurements were made at 60 minute intervals.

In tetraplegics urine output was low with increased osmolality compared to normal subjects and paraplegics, despite a similar water load. Similar observations have been made in patients with chronic autonomic failure after a water load (Wilcox, 1983). This may represent increased secretion or sensitivity to hormones such as vasopressin which regulate water reabsorbtion. After recumbency urine output rose in tetraplegics. There was a small statistically insignificant rise in paraplegics. In tetraplegics the redistribution of extracellular fluid volume with expansion of central blood volume after recumbency, accompanied by an elevation in blood pressure may have inhibited release of vasopressin; these are some of the factors which may have contributed to the diuresis.

Sodium excretion was low in tetraplegics compared to normal subjects. Potassium excretion however was increased after recumbency. The retention of sodium with kaliuresis in tetraplegics may be partly explained by high levels of mineralocortcoids. Tetraplegics are able to increase reflex sympathetic activity via the isolated spinal cord in response to visceral stimuli from the bladder, bowel or skin (Mathias et al., 1983). In these patients the capability to activate spinal sympathetic stimulation may have influenced tubular sodium reabsorption reducing urinary excretion, as demonstrated experimentally (Gottschalk, 1979).

The reasons for increased sodium and potassium excretion in paraplegics at the start of the study with a gradual fall, apparently independent of postural change are unclear. Whether this is related to diurnal changes particular to this group, increased dietary intake of sodium and potassium, or a tubular defect, or incomplete bladder emptying is uncertain. Studies on controlled diets with 24 hour observations will be necessary to answer these questions.

Our studies indicate that recumbency induces a diuresis in tetraplegics but not in paraplegics or normal subjects. It is possible that these effects either at night or during prolonged recumbency due to illness would result in a diuresis and weight loss with reduction of extracellular fluid volume which may increase their susceptibility to postural hypotension. Therapeutic measures in tetraplegics such as head-up tilt may stimulate renin and subsequently aldosterone release; this in addition to other factors including vasopressin release (Sved et al., 1985; Poole et al., 1987) may induce sodium and water retention and plasma volume expansion and subsequently improve low morning blood pressure and symptoms of postural hypotension (Mathias and Frankel, 1983).

\section{References}

Gotтschalk CW 1979 Renal nerves and sodium excretion. Annual Review of Physiology 41:229-240. 
Mathias CJ, Christensen NJ, Corbett JL, et al. 1975 Plasma catecholamines, plasma renin activity and plasma aldosterone in tetraplegic man, horizontal and tilted. Clinical Science and Molecular Medicine 49:291-299.

Mathias CJ, Christensen NJ, Corbett JL, et al. 1976 Plasma catecholamines during paroxysmal neurogenic hypertension in quadriplegic man. Circulation Research 39:204-208.

Mathias CJ, Christensen NJ, Frankel HL, et al. 1980 Renin release during head-up tilt occurs independently of sympathetic nervous activity in tetraplegic man. Clinical Science 59:251-256.

Mathias CJ, Frankel HL 1983 Autonomic failure in tetraplegia. In: Bannister R (ed) Autonomic Failure: a textbook of clinical disorders of the autonomic nervous system. Oxford University Press, Oxford, pp 453-488.

Mathias CJ, Fosbraey P, DaCosta DF, et al. 1986 The effect of desmopressin on nocturnal polyuria, overnight weight loss, and morning postural hypotension in patients with autonomic failure. British Medical fournal 293:353-354.

Poole CJM, Williams TDM, Lightman SL, et al. 1987 Neuroendocrine control of vasopressin secretion and its effect on blood pressure in subjects with spinal cord transection. Brain 110:727-735.

Syed AF, MCDOWEll FH, Blessing WW 1985 Release of antidiuretic hormone in quadriplegic subjects in response to head-up tilt. Neurology, Cleveland 35:78-82. 